\title{
Contexto escolar: práticas educativas do professor, comportamento e habilidades sociais infantis
}

\author{
Alessandra Turini Bolsoni-Silva \\ Universidade Estadual Paulista - SP \\ Maria Luiza Mariano \\ Universidade Estadual Paulista - SP \\ Sonia Regina Loureiro \\ Universidade de Sao Paulo - SP \\ Caroline Bonaccorsi \\ Universidade Estadual Paulista - SP
}

\section{Resumo}

Práticas educativas, problemas de comportamento e habilidades sociais infantis são questões que têm sido pouco estudadas no contexto escolar. Objetivou-se comparar as práticas educativas de professores do ensino regular e do especial com os comportamentos infantis em grupos diferenciados por problemas de comportamento e pelo sexo das crianças. Participaram quinze professoras e vinte e oito alunos (seis a nove anos), distribuídos em dezoito do ensino regular (infantil e fundamental) e dez do ensino especial. Procedeu-se à avaliação com os professores por meio de um questionário (Q-RSH-Pr) e de entrevista semiestruturada (RE-HSE-Pr). Verificou-se, com diferenças significativas, que os meninos e as crianças com problemas de comportamento apresentaram mais dificuldades e suas professoras relataram utilizar mais práticas negativas de educação, e que as meninas apresentaram mais habilidades sociais. Não se encontraram diferenças nas comparações entre o ensino regular e o especial. A comparação entre as práticas educativas dos professores e os comportamentos das crianças no contexto escolar é útil para intervenções.

Palavras-chaves: Educação, distúrbios do comportamento, habilidades sociais.

\section{Teacher's educational practices and child behavior: the influence of multiple variables}

\begin{abstract}
Educational practices, behavior problems and children's social skills have been more studied in family context than in school context. This study aimed to compare, from the verbal report, educational practices of regular and special teacher in relation to children's behavior, concerning groups differentiated by reference indication of behavior problems in the classroom by the teacher and sex of the children. This study surveyed fifteen teachers and twenty-eight students (6-9 years), who were grouped in regular teaching $(n=18$, kindergarten, elementary) and especial teaching $(n=10)$. The assessment with teachers was proceeded by using a questionnaire (Q-RSH-Pr) and a semi-structured interview (RE-HSE-Pr). We observed that there is a significant difference: boys as well as children with behavior problems presented more difficulties than others. Their teachers revealed that they use more negative

Keywords: Education, behavior disorders, social skills.
\end{abstract}

\section{La práctica educativa del profesor y comportamiento infantil: la influencia de múltiplas variables}

\begin{abstract}
Resumen
Prácticas educativas, problemas de comportamiento y habilidades sociales infantiles han sido poco estudiados en el contexto escolar. El estudio tuvo el objetivo de comparar prácticas educativas escolares positivas y negativas; el repertorio conductual respectivo a habilidades sociales e indicadores de problemas de comportamiento; de niños de educación especial y regular, diferenciando grupos de niños que tengan o no problemas de comportamiento y, entre niños y niñas. Participaron de esta investigación 15 profesoras y sus alumnos distribuidos en educación regular (infantil, básica) y especial. La evaluación se realizó con profesores por medio de cuestionario y entrevista semi-estructurada. Los grupos de educación regular y especial no presentaron diferencias en las comparaciones de grupo, los niños y las niñas indicadas con problemas de comportamiento presentaron más problemas de conducta y sus profesoras informaron el uso de más prácticas negativas de educación, las niñas presentaron más
\end{abstract}


habilidades sociales lo que sugiere la necesidad de intervención y nuevos estudios.

Palabras clave: Educación, trastornos de la conducta, habilidades sociales.

\section{Introdução}

Os comportamentos das crianças são complexos e multideterminados, e para o seu entendimento é necessário avaliar o contexto em que ocorrem. Neste sentido, destaca-se que no ambiente familiar as práticas educativas podem tanto promover habilidades sociais como favorecer o surgimento de problemas de comportamento. O ambiente escolar, quando comparado ao familiar, tem sido menos investigado no que se refere às práticas educativas, e o repertório infantil tem justificado estudos neste sentido, embora queixas já estejam bem documentadas.

No contexto escolar, Del Prette e Del Prette (2001), tendo conduzido estudo com uma amostra de professores de uma escola pública, referiram que mais da metade dos professores relatou acontecimentos semanais de conflitos interpessoais como ameaças, xingamentos, agressões físicas, gritos e discussões entre os alunos, além de outras ocorrências diárias envolvendo, em média, cinco a seis alunos agressivos em cada sala. Referiram também pouca efetividade nas tentativas de manejo dessas situações. Por outro lado, todas as habilidades das crianças foram avaliadas como superiores à média, especialmente habilidades sociais como ter boas maneiras, cooperar, compartilhar, desculpar-se, ouvir o outro, pedir favor ou ajuda, fazer perguntas e a elas responder, sendo as classificadas como as mais baixas atribuídas às habilidades de enfrentamento em situações de conflito potencial, como expressar desagrado, corrigir informação, negociar e discordar. Os autores apontaram, a partir dos resultados obtidos, que os professores têm uma compreensão equivocada das habilidades sociais, restrita às habilidades de comunicação e de civilidade, em detrimento de habilidades de enfrentamento e de resolução de problemas das crianças. Então eles sugerem, para os professores, um treinamento sobre manejo dos comportamentos de seus alunos e investimentos no ensino de habilidades sociais infantis, além de habilidades de enfrentamento em situações conflituosas dentro do contexto escolar.

No contexto familiar, a influência das práticas educativas no surgimento e manutenção de problemas de comportamento já está bastante documentada na literatura (Bolsoni-Silva \& Marturano, 2007, 2008; Ferreira \& Marturano, 2002; D’Ávila-Bacarji, Marturano, \& Elias, 2005; Rosa, Garcia, Domingos, \& Silvares, 2001; Weber, Prado, Viezzer, \& Bandenburg, 2004). Bolsoni-Silva, Villas Boas, Romera e Silveira (2010) encontraram a mesma tendência nas interações e repertórios de famílias de crianças com necessidades educacionais especiais (de linguagem e auditiva), ou seja, as práticas positivas foram associadas às habilidades sociais infantis positivas, e as práticas negativas, aos problemas de comportamento.
Sabe-se que mães e professores podem julgar diferentemente os comportamentos das crianças (Achenbach, Mcconaughy, \& Howell, 1987; Bolsoni-Silva, Marturano, Figueiredo, \& Manfrinato, 2006; Feitosa, 2003; Gagnon, Vitaro, \& Tremblay, 1992; Satake, Yoshida, Yamashita, Kinukawa, \& Takagishi, 2003; Kumpulainen e cols., 1999), pois seus ambientes são distintos, apresentando demandas e exigências próprias, o que justifica avaliações em ambos os contextos para melhor compreender as interações estabelecidas entre os adultos e as crianças e os comportamentos das crianças.

Quanto às relações entre práticas educativas e comportamentos infantis no contexto escolar, os estudos são mais escassos. As práticas educativas do professor são mediadas pela cultura, pelo contexto, pela singularidade da história de vida e pela relação estabelecida (Del Prette \& Del Prette, 2001). Bolsoni-Silva e cols. (2010), em revisão de literatura (192 resumos publicados entre o ano de 1986 e junho de 2006) sobre intervenções para reduzir ou prevenir problemas de comportamento, encontraram que apenas $4,2 \%$ dos estudos incluíam avaliação e intervenção com professores apesar de inúmeros estudos, de modo geral, terem ressaltado a importância das práticas positivas do professor na promoção de habilidades sociais e na redução de problemas de comportamento.

Pesquisas no contexto escolar têm atestado a importância da comunicação na prevenção de problemas de comportamento (Howie, Lukacs, Pastor, Reuben, \& Mendola, 2010), no estabelecimento de regras (Gomes, 2003), no treino em resolução de problemas (Allen \& Blackston, 2003), na identificação e reforçamento de comportamentos esperados (Barnett, Bauer, Ehrhardt, Lentz, \& Stdlar, 1996), no oferecimento de suporte (Greene \& Ollendick, 1993), no estabelecimento de estratégias de manejo positivas (Webster-Stratton, Reid, \& Stoolmiller, 2008) e no envolvimento com a família (Webster-Stratton e cols., 2008). Na opinião de muitos professores, seria ideal usar a criatividade e a amizade nas interações estabelecidas com os alunos (Oliveira \& Wechsler, 2002). O estudo de Reis e Camargo (2008), ao entrevistar alunos com TDAH, deixa clara a importância de os professores identificarem precocemente as dificuldades dessas crianças, de modo que possam propor atividades estimulantes e diferenciadas para os alunos, garantindo, assim, a aprendizagem de todos. Tais atividades poderiam prevenir problemas de comportamento, pois as crianças seriam capazes de realizá-las com menos dificuldades e de maneira lúdica.

As práticas educativas positivas dos professores podem ser detalhadas com base nos estudos de Coolahan, Fantuzzo, Mendez, \& McDermott (2000), Del Prette e Del Prette (2002), Gomes (2003), Vila (2005) e Zanotto (2000) como assinalado a seguir. Além do comunicar-se efetivamente com o aluno (olhar para os alunos, falar, ouvir, iniciar comunicação, buscar aproximação, responder perguntas), outros comportamentos são também importantes, como: a) elogiar e ser afetivo (elogiar, expressar sentimentos positivos, dar feedback positivo a comportamentos esperados e agradecer a elogios, apoiar o aluno apesar do seu erro e encorajá-lo a novas tentativas, ser amistoso com o aluno e demonstrar reciprocidade 
quando o aluno é afetuoso, promover situações lúdicas); b) fazer e receber críticas, ouvir e expressar opiniões e alterar o próprio comportamento; c) propor situações-problema, estabelecer regras e incentivar seu cumprimento.

Castro e Bolsoni-Silva (2008) afirmam que o professor, enquanto mediador das relações estabelecidas na sala de aula, pode manter, fortalecer ou até desestimular comportamentos ligados à interação criança-criança e criança-professor, influenciando tanto os aspectos acadêmicos quanto os sociais. Os resultados desse estudo, feito a partir de observação direta, apontaram que os professores incentivavam bons comportamentos das crianças e essas demonstravam contentamento; no entanto, quando as crianças tinham comportamentos que os professores desaprovavam, eles agiam com agressividade (gritando, por exemplo), e nesses momentos as crianças também agrediam. Verificou-se que tanto as crianças como os professores tinham repertório para interagir positivamente em momentos que não estavam relacionados a estabelecer limites, pois nesses momentos tanto crianças como professores agiam com agressividade.

$\mathrm{Na}$ mesma direção, Fonseca (2012), ao descrever estudos de casos de crianças com e sem problemas de comportamento nos ambientes escolar e familiar, encontrou que: a) as professoras identificaram um número maior de comportamentos problemáticos em comparação com as famílias; b) as crianças com problemas de comportamento também apresentavam déficits de desempenho acadêmico a partir do TRF; c) todas as crianças apresentavam bom escore de habilidades sociais; d) todos os familiares apresentaram déficits de práticas positivas e muitas práticas negativas ao educar as crianças, mas essas últimas destacavam-se nas interações com as crianças classificadas como clínicas para problemas de comportamento; e) diferentemente, as professoras apresentavam mais práticas positivas que negativas na forma de interagir com os alunos, principalmente práticas que envolviam comunicação e negociação, aparecendo com menos frequência comportamentos de expressão de afeto/ sentimentos positivos; f) as professoras relataram usar mais práticas positivas e menos negativas com as crianças sem problemas de comportamento, ocorrendo o contrário com as crianças com problemas de comportamento.

Mariano (2011), ao investigar práticas educativas e comportamentos infantis junto a 16 professoras e seus alunos, encontrou que elas apresentavam práticas positivas de interação, sobretudo as de comunicação, mas as qualidades dessas práticas eram diferentes conforme identificavam as crianças com e sem problemas de comportamento. Para as crianças que elas consideravam problemáticas, o conteúdo da comunicação era corrigir os comportamentos, e com o grupo considerado sem problemas, elas conversavam sobre assuntos de interesse das crianças. Notou-se que elas relataram agir de maneira diferente conforme a criança, mas eram mais positivas com as crianças que apresentavam os comportamentos esperados.

Segundo Bandeira e Gaglia (2006), para serem bem-sucedidas, as crianças precisam ter comportamento assertivo, pois esse comportamento contribui para melhorar a comunicação interpessoal, expressar sentimentos e necessidades. Por outro lado, cabe ao professor identificar a importância de tais comportamentos para o desenvolvimento da criança, buscando estimulá-los mesmo em situações que envolvam estabelecer limites.

Dada a complexidade das demandas do ambiente escolar envolvendo o ensino formal e o desenvolvimento pessoal das crianças quanto à socialização, pode-se pensar que os professores se beneficiariam da aprendizagem e aprimoramento das suas práticas educativas quanto ao que pode auxiliar em suas ações dos psicólogos escolares em suas ações. Daí a importância de uma adequada formação dos professores, especialmente no que tange a lidar com as diferenças e com novas estratégias para o ensino de conteúdo acadêmico e social. Nessa direção, o estudo de Longarezi e Alves (2009) foi capaz de promover discussões sobre a importância de os professores serem afetivos com seus alunos e de ensiná-los a ser autônomos.

Almeida, Alves, Neves, Silva e Pedroza (2007), a partir de entrevistas conduzidas com 30 professores, verificaram que eles pouco se lembravam do que haviam aprendido sobre psicologia, o que indica a necessidade de rever os cursos de licenciatura; mas também alertam que tais conteúdos, imprescindíveis para o ensino acadêmico e social dos alunos, dificilmente serão utilizados de maneira eficiente pelo professor em sala de aula, o que poderá maximizar problemas de interação com os alunos. Respaldando esses achados, Paiva e Del Prette (2009) verificaram que os professores considerados facilitadores no processo ensino-aprendizagem acreditavam que o conteúdo acadêmico estava relacionado também ao repertório de habilidades sociais.

Uysal e Ergenekon (2010), Para sugerir habilidades sociais em ambientes naturais e ensiná-las aos indivíduos que apresentam deficiências sociais, Uysal e Ergenekon fizeram um estudo com professores para identificar suas práticas de ensino sobre habilidades sociais em Educação Especial. Os resultados demonstraram que os professores estavam tendo sérios problemas e inadequações em relação ao ensino dessas habilidades. Os autores concluiram que, para garantir o aprendizado de crianças com necessidades educacionais especiais, o ensino das habilidades sociais precisa ser conduzido de forma sistemática. Com esse objetivo os professores participaram de uma intervenção para aprenderem a ensinar habilidades sociais a seus alunos. Os autores verificaram que o repertório de habilidades sociais das crianças aumentou após esse ensino aos professores. Com sentido semelhante, destaca-se o estudo de Del Prette, Del Prette, Garcia, Bolsoni-Silva e Puntel (1998), que ensinou aos professores diversas habilidades sociais para melhorar suas interações com as crianças.

Com relação à interação entre pais e filhos, Bolsoni-Silva e Loureiro (2011) propuseram a seguinte classificação para as habilidades sociais educativas parentais (HSE-P): comunicação (conversar, perguntar); expressão de sentimentos e enfrentamento (expressar sentimentos positivos, negativos e opiniões, demonstrar carinho, brincar); e estabelecimento de limites (identificar comportamentos social- 
mente habilidosos e não habilidosos, estabelecer regras, ter consistência, cumprir promessas, identificar erros e pedir desculpas). Tais categorias, elaboradas a partir do relato espontâneo de mais de 200 cuidadores, permitiram elaborar e validar um instrumento, denominado de Roteiro de Habilidades Sociais Educativas Parentais (RE-HSE-P), que avalia funcionalmente a frequência e a qualidade de práticas educativas positivas, negativas, habilidades sociais infantis e problemas de comportamento (Bolsoni-Silva, Loureiro, \& Marturano, 2011). Dadas as características das interações estabelecidas entre professores e alunos, guardadas as peculiaridades do contexto, pode-se pensar que, de um modo geral, as categorias amplas, relativas às práticas educativas (positivas e negativas), problemas de comportamento e habilidades sociais infantis, além de variáveis contextuais, possam também ser avaliadas no ambiente escolar de forma a identificar interações promotoras ou não do desenvolvimento das crianças.

As relações entre habilidades sociais e problemas de comportamento não apresentam resultados unívocos. Alguns estudos identificaram relação inversa (Bolsoni-Silva \& Loureiro, 2011; Cia \& Barham, 2009) e outros não, como o estudo de Pierson, Carter, Lane, e Glaeser (2004) e Fonseca (2012). Pierson e cols. (2004) investigaram esse tema com 90 estudantes na transição entre dois períodos escolares quanto aos repertórios de habilidades sociais e de problemas de comportamento no que refere ao conceito de autodeterminação. Os autores encontraram relação positiva entre habilidades sociais e autodeterminação, mas não quanto a problemas de comportamento.

Outro ponto a ser destacado diz respeito ao sexo. Geralmente os meninos são mais indicados para atendimento psicológico (Massola \& Silvares, 1997), embora haja evidências de que eles não apresentam mais problemas que as meninas (Marturano, Parreira, \& Benzoni, 1997). Tais indicações podem estar relacionadas à maior frequência de problemas de externalização (agressividade e desobediência, por exemplo) em meninos e de internalização em meninas (timidez, tristeza, por exemplo) (Patterson, Reid, \& Dishion, 2002), sendo que esses últimos causam menor desconforto para os agentes educativos.

A literatura não é consensual sobre a relação entre os comportamentos de habilidades sociais e problemas de comportamento. Diversos estudos descrevem ou os comportamentos das crianças ou as práticas educativas dos professores, mas poucos são os que buscam avaliar concomitante esses comportamentos, ou seja, as interações sociais estabelecidas entre professores e alunos dos ensino regular e especial. É por exte contesto que se justifica o presente estudo, com delineamento transversal de comparações entre grupos de crianças diferenciados pelo sexo e pela presença de problemas de comportamento.

Teve-se como objetivo comparar as práticas educativas de professores do ensino regular e do especial (positivas e negativas) com os comportamentos infantis relativos às habilidades sociais e a problemas de comportamento das crianças. Estas foram divididas em grupos diferenciados pela presença de indicação, pelo professor, de problema de comportamento, e pela diferenciação do sexo das crianças.

\section{Método}

\section{Participantes}

Foram participantes quinze professoras (cinco professoras do ensino especial, cinco do fundamental e cinco do infantil) e 28 alunos (dezoito do ensino regular e dez do ensino especial).

Caracterizando as professoras quanto ao tempo de docência, verificou-se que este variou entre cinco e vinte anos de exercício na rede municipal de ensino de uma cidade do Centro-Oeste Paulista, sendo que para duas delas faltavam apenas seis meses para aposentadoria (média: sete anos; desvio padrão: sete anos). Suas idades variavam entre 23 e 50 anos (média de 33,56 anos) e todas tinham formação em Pedagogia.

Os critérios de seleção dos professores foram: concordar espontaneamente em participar da pesquisa, ter vínculo efetivo com a rede municipal de ensino, dar aulas para os alunos do $1^{\circ}$ ano e possuir tempo de serviço entre três e dez anos. Foram excluídos professores substitutos e/ ou eventuais na escola participante e os que não assinaram previamente o termo de consentimento para participar da pesquisa.

Por sua vez, os alunos tinham idade entre seis e nove anos. Quinze deles apresentavam problemas de comportamento e treze não os apresentavam, de acordo com a informação verbal das professoras, quando Ihes foi solicitado que indicassem crianças que, na opinião delas, apresentavam ou não comportamentos tidos como problemas na sala de aula. Quanto ao sexo, doze crianças eram do sexo masculino e dezesseis do sexo feminino. Para verificar se os grupos de crianças distribuídas entre ensino regular ( $n=$ 10) e especial $(n=18)$ eram homogêneos quanto ao sexo, foi feita a análise estatística Crosstabs e não foram encontradas diferenças estatisticamente significativas entre os grupos ( $p=0,208$ para meninos e $p=0,143$ para meninas).

As dificuldades apresentadas pelas crianças do ensino especial foram documentadas por avaliações sistemáticas médicas e educacionais: síndrome de Down $(n=1)$, autismo $(n=1)$, distúrbios da fala e de comportamento $(n=$ 2 ); dificuldades de aprendizagem $(n=3)$ e dificuldades de socialização - timidez ou hiperatividade $(n=3)$. As outras três crianças que integravam a amostra do ensino especial não estavam acompanhando as salas regulares e foram encaminhadas para a Sala de Recursos (ensino especial). 
Destaca-se que todas as crianças do ensino especial também frequentavam o ensino regular. Todas as crianças do ensino infantil tinham seis anos e todas as do ensino fundamental estavam com sete anos. As idades das crianças no ensino especial eram: seis anos $(n=4)$, sete anos $(n=3)$, oito anos $(n=2)$ e nove anos $(n=1)$.

\section{Local}

A coleta de dados ocorreu em 10 escolas da rede municipal de uma cidade do Centro-Oeste do Estado de São Paulo.

\section{Instrumentos}

O Roteiro de Entrevista de Habilidades Sociais Educativas para Professores - RE-HSE-Pr (Adaptação de Bolsoni-Silva e cols., 2011) é um roteiro de entrevista composto por questões que se referem à forma como o(a) professor(a) se comporta com relação a seu aluno(a), e vice-versa. Não há respostas certas ou erradas, pretende-se apenas conhecer sobre o relacionamento entre o professor e o aluno. Ao final são investigados seus níveis socioeconômicos e educacionais. Nas questões há perguntas sobre: a) frequência com que aparece o comportamento mencionado; e b) características desses comportamentos. Quanto à frequência, o respondente deverá escolher apenas uma das alternativas: frequentemente, se o comportamento acontecer muitas vezes durante a semana; algumas vezes, se o comportamento acontecer poucas vezes durante a semana ; e quase nunca ou nunca, se o comportamento aparecer a cada quinze dias ou um mês ou mais. O instrumento permite obter escores sobre as práticas educativas (positivas e negativas), problemas de comportamento e habilidades sociais infantis, além de variáveis contextuais. O alpha para os 78 itens que compõem o instrumento, na versão professores, foi de 0,724.

O Questionário de Respostas Socialmente Habilidosas - versão professores (QRSH-PR) possui uma lista de comportamentos socialmente habilidosos, constando de vinte e quatro itens, com uma escala de três pontos (2 = se aplica; 1 = se aplica em parte; 0 = não se aplica), cujos escores são somados, fornecendo o escore total da criança avaliada. Estudos sobre as propriedades psicométricas do instrumento realizados por Bolsoni-Silva, Marturano e Loureiro (2009) indicaram boa consistência interna e validades de constructo, concorrente e preditiva. $\mathrm{O}$ instrumento permite organizar os dados em três fatores: Sociabilidade e Expressividade Emocional (tem relações positivas, mostra interesse pelos outros, faz amigos, expressa frustração, comunica-se, expressa desejos, expressa direitos, expressa carinho, brinca com colegas, usualmente fica de bom humor, faz elogios, cumprimenta, interage de forma não verbal, participa de grupos); Iniciativa Social (toma a palavra, expressa opiniões, participa de temas de discussão, toma iniciativas, negocia e convence, presta ajuda); e Busca de Suporte (procura atenção, faz pedido, faz perguntas).

\section{Procedimentos de coleta de dados}

Inicialmente o projeto foi encaminhado ao comitê de ética da universidade em que foi realizada a pesquisa, o qual concedeu aprovação (Processo no. 2567/46/01/09). Na sequência, foi encaminhada à Secretaria da Educação um pedido de autorização para a execução da pesquisa em escolas sob sua jurisdição, pedido que também foi deferido.

$\mathrm{Na}$ escola as professoras participaram de uma reunião para a apresentação do projeto e resolução de dúvidas. Aquelas que preencheram os requisitos e aceitaram participar assinaram o Termo de Consentimento Livre e Esclarecido (TCLE) e indicaram uma criança que consideravam ter problemas de comportamento e uma que consideravam sem problemas de comportamento, ambas da turma em que lecionavam. Tal medida foi tomada visando ter nas três amostras equilíbrio quanto à indicação ou não de problemas de comportamento para cada uma das professoras. As professoras responderam aos instrumentos em horário previamente combinado e nos seus horários de reunião (HTPC) ou extraclasse, em locais de sua preferência dentro da escola. As entrevistas foram gravadas com seu consentimento.

\section{Procedimentos de tratamento e análise de dados}

Os dados foram categorizados conforme instruções próprias dos instrumentos e submetidos à análise estatística (SPSS). Foram conduzidos testes não paramétricos para as comparações de grupos quanto ao tipo de ensino, a ter ou não indicação escolar de problema de comportamento e quanto ao sexo da criança (Testes Mann-Whitney).

\section{Resultados}

Essa seção apresenta primeiramente os resultados de comparações dos grupos diferenciados pelo tipo de ensino destacando as habilidades sociais e as práticas educativas, como mostra a tabelas 1 .

Verifica-se que nas comparações dos grupos ensino especial e regular não foram observadas diferenças estatisticamente significativas quanto aos comportamentos das professoras e crianças.

A análise feita pelas professoras das comparações relativas aos grupos diferenciados pela indicação de crianças com problemas de comportamento e de crianças sem problemas de comportamento são apresentadas na tabela 2 .

Notou-se que, em relação ao grupo com indicador escolar de problemas de comportamento, as professoras relataram utilizar mais práticas negativas de educação, o que traz um impacto para o total negativo, que é a soma conjunta das práticas negativas e dos problemas de comportamento. Por outro lado, em relação ao grupo sem problemas de comportamento verificou-se a presença de mais habilidades sociais 
Tabela 1. Resultados na comparação entre os Grupos Ensino Especial (EE) e Ensino Regular (ER), no que se refere às classificações do RE-HSE-Pr e do QRSH-Pr(TesteMann-Whitney).

\begin{tabular}{|c|c|c|c|}
\hline Categorias dos Instrumentos & $\begin{array}{l}\text { Ensino Especial } \\
\qquad(n=10)\end{array}$ & $\begin{array}{l}\text { Ensino Regular } \\
\qquad(\mathrm{n}=18)\end{array}$ & $p$ \\
\hline \multicolumn{4}{|c|}{ Médias (DesviosPadrão) } \\
\hline \multicolumn{4}{|c|}{ RE-HSE-Pr } \\
\hline Habilidadessociaisinfantis & $11,10(3,07)$ & $12,40(4,74)$ & 0,879 \\
\hline Habilidade social educativa & $8,80(3,58)$ & $9,30(3,67)$ & 0,820 \\
\hline Práticanegativa & $7,90(3,73)$ & $8,45(7,13)$ & 0,705 \\
\hline Problema de comportamento & $0,90(1,20)$ & $2,30(2,05)$ & 0,066 \\
\hline Contexto & $11,30(4,47)$ & $11,05(4,10)$ & 0,970 \\
\hline Total positivo & $31,20(7,35)$ & $32,75(9,63)$ & 0,820 \\
\hline Total negativo & $8,80(4,69)$ & $10,75(8,28)$ & 0,910 \\
\hline \multicolumn{4}{|c|}{ QRSH-Pr } \\
\hline Fator 1 & $20,40(6,36)$ & $20,05(7,73)$ & 0,939 \\
\hline Fator 2 & $8,30(3,65)$ & $8,28(2,99)$ & 0,790 \\
\hline Fator 3 & $5,10(1,29)$ & $5,05(1,35)$ & 0,775 \\
\hline Total & $35,10(9,78)$ & $34,44(9,15)$ & 0,595 \\
\hline
\end{tabular}

Tabela 2. Resultados na comparação entre osGrupos Problemas de comportamento e semproblemas de comportamento no que se refere às classificações do RE-HSE-Pr e do QRSH-Pr (Testet de Student).

\begin{tabular}{|c|c|c|c|}
\hline Categorias dos Instrumentos & $\begin{array}{c}\text { Problema } \\
(n=15)\end{array}$ & $\begin{array}{l}\text { Semproblema } \\
\qquad(\mathrm{n}=13)\end{array}$ & $p$ \\
\hline \multicolumn{4}{|c|}{ Médias (DesviosPadrão) } \\
\hline \multicolumn{4}{|c|}{ RE-HSE-Pr } \\
\hline Habilidadessociaisinfantis & $11,06(3,77)$ & $13,00(4,66)$ & 0,260 \\
\hline Habilidade social educativa & $9,94(3,57)$ & $8,21(3,51)$ & 0,203 \\
\hline Práticanegativa & $11,50(6,00)$ & $4,57(3,88)$ & 0,003 \\
\hline Problema de comportamento & $2,31(2,09)$ & $1,29(1,59)$ & 0,141 \\
\hline Contexto & $10,25(3,19)$ & $12,14(4,96)$ & 0,234 \\
\hline Total positivo & $31,25(7,02)$ & $33,36(10,72)$ & 0,771 \\
\hline Total negativo & $13,81(7,30)$ & $5,86(4,40)$ & 0,003 \\
\hline \multicolumn{4}{|c|}{ QRSH-Pr } \\
\hline Fator 1 & $17,33(7,44)$ & $23,46(5,36)$ & 0,023 \\
\hline Fator 2 & $9,20(2,48)$ & $7,23(3,63)$ & 0,152 \\
\hline Fator 3 & $5,60(0,51)$ & $4,46(1,66)$ & 0,071 \\
\hline Total & $33,27(9,13)$ & $36,31(9,38)$ & 0,474 \\
\hline
\end{tabular}


Tabela 3.Resultados na comparação entre osGrupos de meninos e de meninas no que se refere às classificações do REHSE-Pr e do QRSH-Pr(Testet de Student).

\begin{tabular}{|c|c|c|c|}
\hline Categorias dos Instrumentos & $\begin{array}{c}\text { Meninos } \\
(n=12)\end{array}$ & $\begin{array}{c}\text { Meninas } \\
(n=16)\end{array}$ & $p$ \\
\hline \multicolumn{3}{|c|}{ Médias (DesviosPadrão) } & \\
\hline Habilidadessociaisinfantis & $10,15(3,58)$ & $13,35(4,29)$ & 0,051 \\
\hline Habilidade social educativa & $9,77(3,72)$ & $8,65(3,52)$ & 0,437 \\
\hline Práticanegativa & $10,85(6,49)$ & $6,29(5,22)$ & 0,065 \\
\hline Problema de comportamento & $2,77(2,13)$ & $1,12(1,41)$ & 0,020 \\
\hline Contexto & $10,85(3,11)$ & $11,35(4,89)$ & 0,674 \\
\hline Total positivo & $30,77(6,77)$ & $33,35(10,20)$ & 0,476 \\
\hline Total negativo & $13,62(7,64)$ & $7,41(5,81)$ & 0,029 \\
\hline \multicolumn{4}{|c|}{ QRSH-Pr } \\
\hline Fator 1 & $18,33(7,54)$ & $21,56(6,76)$ & 0,191 \\
\hline Fator 2 & $8,33(3,17)$ & $8,25(3,28)$ & 0,981 \\
\hline Fator 3 & $5,33(1,15)$ & $4,88(1,41)$ & 0,349 \\
\hline Total & $32,75(9,67)$ & $36,13(8,88)$ & 0,352 \\
\hline
\end{tabular}

condizentes com o Fator 1 do QRSH-Pr - Sociabilidade e Expressividade Emocional - envolvendo os seguintes componentes: tem relações positivas, mostra interesse pelos outros, faz amigos, expressa frustração, comunica-se, expressa desejos, expressa direitos, expressa carinhos, brinca com colegas, usualmente fica de bom humor, faz elogios, cumprimenta, interage de forma não verbal, participa de grupos.

As comparações relativas aos grupos diferenciados pelo sexo das crianças são apresentadas na tabela 3 .

Verificou-se, com diferenças significativas, que as meninas foram avaliadas pelos professores no RE-HSE-Pr como mais habilidosas que os meninos e estes como mais problemáticos quanto ao comportamento e com maior escore de total negativo que aquelas. Interessante notar que tanto os meninos como as meninas, segundo a avaliação das professoras, apresentaram repertório de habilidades sociais (QRSH-Pr), e as professoras relataram usar habilidades sociais educativas para interagir com as crianças de ambos os sexos.

\section{Discussão}

Considera-se que os resultados da presente pesquisa ajudaram a caracterizar categorias de comportamentos da interação professor-aluno, diferenciando modalidades de ensino, crianças com problemas de comportamento e meninos e meninas.

Interessante notar que as comparações entre os grupos de crianças do ensino especial e regular não diferiram nem no tocante às práticas educativas (positivas e negativas) nem quanto ao repertório comportamental das crianças (habilidades sociais e problemas de comportamento). Dessa forma, com base nesses dados e dentro dos seus limites, não se pode afirmar que as crianças que frequentam salas de recurso (Ensino Especial) apresentam mais problemas de comportamento, o que sugere a não associação entre necessidades educacionais especiais e problemas de comportamento. Comparativamente a dados obtidos no contexto familiar, pode-se relatar o estudo de Bolsoni-Silva e cols. (2010), o qual demonstrou que os pais de crianças com deficiência auditiva ou de linguagem, quando interagiam de maneira positiva, promoviam habilidades sociais nos filhos, e quando utilizavam frequentemente práticas negativas, estimulavam problemas de comportamento. Tais relações são bem documentadas no caso do contexto familiar (Patterson, DeBaryshe, \& Ramsey, 1989; Patterson e cols., 2002), mesmo na ausência de alguma necessidade educativa especial. Dada a disponibilidade de poucos dados que avaliem concomitantemente práticas dos professores e comportamentos das crianças, ao se fazer a comparação com dados do contexto familiar pode-se especular que o mesmo padrão obtido 
com os pais parece também ocorrer com os professores no contexto escolar, evidenciando que as práticas educativas e os comportamentos das crianças parecem independer da existência ou não de uma necessidade especial associada.

As crianças indicadas como problemáticas quanto ao comportamento de fato apresentaram maior frequência de comportamentos negativos, confirmando a suposição das professoras. Este resultado sugere que as professoras são boas avaliadoras dos comportamentos das crianças, o que está concordância com o constatado em outros estudos (Gresham, Noell, \& Elliott, 1996).

Destaca-se ainda que embora os resultados sejam concordantes quanto à associação dos problemas de comportamento a práticas negativas de educação (Patterson e cols., 2002; Weber e cols., 2004), não se pode afirmar que os problemas de comportamento são mais frequentes quando há baixa frequência de habilidades sociais educativas (práticas positivas). Tais associações já foram demonstradas em estudos conduzidos a partir do contexto familiar (Bolsoni-Silva \& Loureiro, 2011) e da escola, em aspectos como o estabelecimento de regras (Gomes, 2003), o treino na resolução de problemas (Allen \& Blackston, 2003), a identificação e reforçamento de comportamentos alvo (Bauer e cols., 1996), o oferecimento de suporte (Greene \& Ollendick, 1993) e o estabelecimento de estratégias de manejo positivas (Webster-Stratton, Reid, \& Stoolmiller, 2008) que são comportamentos que poderiam ser associados ao repertório de habilidades sociais educativas do professor.

Não obstante, com base nos estudos de Del Prette e Del Prette (2001), Castro e Bolsoni-Silva (2008), Mariano (2011) e Fonseca (2012), pode-se supor que, se as crianças possuem um bom repertório de habilidades sociais, estas podem não estar sendo devidamente estimuladas no contexto escolar, o que pode estar favorecendo as manifestações de agressividade e desobediência (consideradas problemas de comportamento) para obter atenção, resolver problemas e livrar-se de tarefas que elas consideram difíceis. Quanto aos professores, embora possuam boas práticas positivas, podem estar falhando no uso contingente a comportamentos que desaprovam, o que ocorre em situações de estabelecimento de limites. Consequentemente, podem estar, inadvertidamente, dando aos alunos exemplo de agressividade, levando-os a pensar que podem usar da agressividade para resolver problemas e obter atenção. Conforme os autores acima mencionados, as professoras são capazes de agir de maneira diferenciada conforme a criança, sugestão dada por Reis e Camargo (2008), mas parecem estar fazendo isto de uma maneira que dificulta mais as interações estabelecidas com as crianças que apresentam comportamentos considerados inapropriados.

De todo modo, o fato de as professoras apresentarem práticas positivas de educação sugere a presença de recursos que podem ser potencializados por intervenções que visem reduzir problemas de comportamento das crianças e práticas educativas negativas dos professores.

Tais afirmativas são concordantes com Uysal e Ergenekon (2010), os quais apontam que as intervenções junto aos professores poderiam ajudá-los a identificar e valorizar comportamentos de habilidades sociais das crianças. Tendo-se em vista o suporte de estudos mencionados (Coolahan e cols., 2000; Del Prette \& Del Prette, 2002; Gomes, 2003; Oliveira \& Wechsler, 2002; Vila, 2005; Zanotto, 2000), considera-se que seria importante também, para a formação continuada dos professores, ajudá-los-los a usar as práticas educativas positivas/habilidades sociais educativas (de comunicação, afeto e expressividade) de que já fazem uso em outros contextos para as situações de estabelecimento de limites. A aprendizagem de novos recursos pedagógicos que tenham como foco a definição clara das regras, tal como se colocam nos jogos e brincadeiras que fazem parte do cotiano das crianças, poderá, de modo criativo, ajudar nas interações no contexto escolar, favorecendo que os professores sejam bons modelos de comportamento de autocontrole e resolução de problemas, podendo estimular os alunos na mesma direção. Por outro lado, a maior clareza por parte dos professores poderá também facilitar a identificação e ajudar as crianças que já apresentam, especialmente na primeira infância, dificuldades de interações sociais.

No que se refere ao repertório de habilidades sociais das crianças, nota-se que parte dele foi mais frequente no grupo sem dificuldade de comportamento, o que está em concordância com os estudos desenvolvidos no contexto familiar por Bolsoni-Silva e Loureiro (2011) e com Cia e Barham (2009). Os itens que diferenciaram os grupos correspondem à subescala do QRSH-Pr (Sociabilidade e Expressividade Emocional - tem relações positivas, mostra interesse pelos outros, faz amigos, expressa frustração, comunica-se, expressa desejos, expressa direitos, expressa carinhos, brinca com colegas, usualmente fica de bom humor, faz elogios, cumprimenta, interage de forma não verbal, participa de grupos); por outro, a medida de habilidades sociais a partir do RE-HSE-Pr no contexto escolar, não diferenciou os grupos com e sem dificuldade de comportamento. Pode-se pensar que as crianças com problemas de comportamento podem ter repertório de habilidades sociais, mas não estariam sendo valorizadas e/ou estimuladas a comportarem-se dessa forma (Del Prette \& Del Prette, 2001), por isso não estariam aparecendo na fala das participantes. Adicionalmente, como demonstrado por Bolsoni-Silva e cols. (2011), o uso de entrevistas e escalas, ainda que ambas mensurem a partir do relato verbal, permite obter dados diferentes e complementares quando o relato é dirigido e não dirigido. De todo modo, esses dados sugerem que é necessário utilizar mais de um instrumento de avaliação e a avaliação em mais de um contexto, de forma a complementar a avaliação dos comportamentos de interesse e ampliar a compreensão sobre os recursos e dificuldades das crianças.

A análise da diferenciação pelo sexo das crianças levou a constatar que os meninos apresentaram mais indicadores de problemas de comportamento, o que esta em concordância com o estudo de Marturano e cols. (1997) e em discordância com o estudo de Patterson e cols. (2002). As professoras relataram utilizar mais práticas negativas com meninos do que com meninas, possivelmente devido ao 
tipo de problema externalizante de comportamento mais frequentemente apresentado por eles em comparação com as meninas, entre as quais predominam os internalizante. Não obstante, nesse estudo os problemas comportamentais não foram categorizados em externalizantes e internalizantes, tendo a afirmativa acima um caráter mais especulativo que demonstrativo. Destaca-se ainda que as meninas apresentaram, segundo suas professoras, mais habilidades sociais, o que pode ter funcionado como comportamento funcionalmente equivalentes (Goldiamond, 2002), mantendo a frequência baixa de comportamentos considerados problemáticos.

Considera-se que o presente estudo, por ter sido conduzido com um pequeno número de participantes, na condição natural em que estes foram identificados e sem um maior rigor quanto à homogeneidade dos grupos, é preciso ser visto como preliminar e exploratório. A principal contribuição dos dados está em ter evidenciado no contexto escolar - portanto, com alta validade ecológica -, comparações entre práticas educativas dos professores e os comportamentos das crianças, campo que carece de dados que possam funcionar como pistas para o manejo das complexas interações estabelecidas. São necessários novos estudos que, com maior número de participantes, controlem variáveis como a sobreposição de características relativas às dificuldades comportamentais, o sexo das crianças e as modalidades de ensino, de modo a caracterizar de forma mais ampla e precisa as práticas educativas dos professores.

\section{Considerações finais}

Dada a escassez da literatura sobre práticas educativas do professor e o repertório infantil avaliados no mesmo contexto, as contribuições deste estudo, ainda que preliminar, abre perspectiva para novos estudos no nosso contexto. Destaca-se que no contexto escolar as práticas negativas de educação mostraram-se relacionadas a mais problemas comportamentais das crianças, tal como já se verificou no ambiente familiar. Problemas de comportamento e habilidades sociais parecem ser comportamentos funcionalmente equivalentes e os meninos parecem ter mais dificuldades de interação social que as meninas. Como desdobramento do estudo, depreende-se dos dados a necessidade de intervenções nas escolas, sobretudo para promover habilidades sociais dos meninos e ajudar os professores a utilizarem menos práticas negativas de educação.

\section{Referências}

Achenbach, T. M., Mcconaughy, S. H., \& Howell, C. T. (1987). Child/ adolescent behavioral and emotional problems: Implications of cross-informant correlations for situational specificity. Psychological Bulletin, 101, 213-232.

Allen, S. J., \& Blackston, A. R. (2003). Training preservice teachers in collaborative problem solving: An investigation of the impact of teacher and student behavior change in real-world settings. School Psychology Quarterly, 18(1), 22-51.

Almeida, R. S., Alves, C. B., Neves, G. N., Silva, L. P., \& Pedroza, R. L. S. (2007). O professor de ensino médio e a psicologia em seu cotidiano escolar Professor de ensino médio-psicologia. Revista Semestral da Associação Brasileira de Psicologia Escolar e Educacional (ABRAPEE), 11(1), 123-132.

Bandeira, M., \& Quaglia, M. A. C. (2006). Comportamento assertivo: relações com ansiedade, lócus de controle e auto-estima (pp. 1746). Em M. Bandeira, Z. A. P. Del Prette \& A. Del Prette (Orgs.), Estudos sobre habilidades sociais e relacionamento interpessoal. São Paulo: Casa do Psicólogo.

Barnett, D. W., Bauer, A. M., Ehrhardt, K. E., Lentz, F. E., \&. Stollar, S. A. (1996). Keystone Targets for Change: Planning For Widespread Positive Consequences. School Psychology Quarterly, 11(2), 95117.

Bolsoni-Silva, A. T., \& Loureiro, S. R. (2011). Práticas educativas parentais e repertório comportamental infantil: comparando crianças diferenciadas pelo comportamento. Paidéia, 21(48), 6171.

Bolsoni-Silva, A. T., \& Marturano, E. M. (2002). Práticas educativas e problemas de comportamento: Uma análise à luz das habilidades sociais. Estudos de Psicologia (Natal), 7(2), 227-235.

Bolsoni-Silva, A. T., \& Marturano, E. M. (2007). A qualidade da interação positiva e da consistência parental na sua relação com problemas de comportamento de pré-escolares. Revista Interamericana de Psicologia, 41(3), 349-358.

Bolsoni-Silva, A. T., \& Marturano, E. M. (2008). Habilidades Sociais Educativas Parentais e problemas de comportamento: Comparando pais e mães de pré-escolares. Aletheia (ULBRA), 27(1), 126-138.

Bolsoni-Silva, A. T., Loureiro, S. R., \& Marturano, E. M. (2011). Problemas de comportamento e habilidades sociais infantis:modalidades de relatos. Psico, 42(3), 347-354.

Bolsoni-Silva, A. T., Loureiro, S. R., \& Marturano, E. M. (2011). Roteiro de Entrevista de Habilidades Sociais Educativas Parentais (REHSE-P). São Paulo: Vetor.

Bolsoni-Silva, A. T., Marturano, E. M., \& Loureiro, S. R. (2009). Construction and validation of the brazilian Questionario de Respostas Socialmente Habilidosas segundo relato de professores (QRSH-PR). Spanish Journal of Psychology, 12(1), 349 - 359.

Bolsoni-Silva, A. T., S. R., Marturano, E. M., Figueiredo, V. A. P., \& Manfrinato, J. W. S. (2006). Habilidades sociais e problemas de comportamento de pré-escolares: comparando avaliações de mães e de professoras. Psicologia: Reflexão e Crítica, 19(3), 460469. 
Bolsoni-Silva. A. T., Villas Boas, A. C. V. B., Romera, V. B., \& Silveira F. F. (2010). Caracterização de programas de intervenção com crianças e/ou adolescentes. Arquivos Brasileiros de Psicologia, 62(1), 104-115

Castro, A. B., \& Bolsoni-Silva, A. T. (2008). Habilidades sociais na educação: relação entre concepções e práticas docentes na educação infantil. Em V. L. M. \& F. Capellini (Org.), Políticas públicas, práticas pedagógicas e ensino-aprendizagem: diferentes olhares sobre o processo educacional. Ed. Bauru : Cultura Acadêmica.

Cia, F., \& Barham, E. J. (2009). Repertório de habilidades sociais, problemas de comportamento, autoconceito e desempenho acadêmico de crianças no início da escolarização. Estudos de Psicologia, 26(1), 45-55.

Coolahan, K., Fantuzzo, J., Mendez, J., \& McDermott, P. (2000). Preschool peer interactions and readiness to learn: relationships between classroom peer play and learning behaviors and conduct. The American Psychological Association, 92(3), 458-465.

D'Avila-Bacarji, Marturano, E. M., \& Elias, L. C. S. (2005) Suporte parental: um estudo sobre crianças com queixas escolares. Psicologia em estudo, 10(1), 107-115

Del Prette, Z. A. P., \& Del Prette, A. (2001). Psicologia das relações interpessoais. Vivências para o trabalho em grupo. Petrópolis, RJ: Vozes.

Del Prette, Z. A. P., \& Del Prette, A. (2002). Avaliação de habilidades sociais de crianças com um inventário multimídia: Indicadores sociométricos associados a frequência versus dificuldade. Psicologia em Estudo, 7(1), 61-73.

Del Prette, Z. A. P., Del Prette, A., Garcia, F. S., Bolsoni-Silva, A. T., \& Puntel, L. P. (1998). Habilidades social do professor em sala de aula: um estudo de caso. Psicologia: Reflexão e Crítica, 11(3), 591-603.

Feitosa, F. B. (2003). Relação família-escola: Como pais e professoras avaliam e reagem ao repertório social de crianças com e sem dificuldades de aprendizagem. Dissertação de Mestrado, Curso de Pós-Graduação em Educação Especial, Universidade Federal de São Carlos, São Paulo.

Ferreira, M. C. T., \& Marturano, E. M. (2002) Ambiente Familiar e os Problemas do Comportamento apresentados por Crianças com Baixo Desempenho Escolar. Psicologia Reflexão e Crítica, 15(1), 35-44.

Fonseca, B. C. R. (2012). Práticas educativas de genitores e professoras e repertório comportamental de crianças do ensino fundamental: estudos de caso. Dissertação de Mestrado, Curso de Pós-Graduação em Psicologia do Desenvolvimento e Aprendizagem, Universidade Estadual Paulista, São Paulo.
Gagnon, C., Vitaro, F., \& Tremblay, R. E. (1992). Parent-teacher agreement on kindergartners' behavior problems: A research note. Journal of Child Psychology and Psychiatry, 33, 1255-1261

Goldiamond. (2002). Toward a constructional approach to social problems: Ethical and constitutional issues raised by Applied Behavior Analysis. Behavior and Social Issues, 11,108-197.

Gomes, M. A. M. A. (2003). A utilização dos jogos na educação: diferentes abordagens. Argumento, $V(10), 117-129$

Greene, R. W., \& Ollendick, T. H. (1993). Evaluation of a multidimensional program for sixth-grader in transition from elementary to middle school. Journal of Community Psychology, $21,162-176$.

Gresham, F. M., Noell, G. H., \& Elliott, S. N. (1996). Teachers as judges of social competence: A conditional probability analysis. School Psychology Review, 25, 108-117.

Howie, L. D., Lukacs S. L., Pastor P. N., Reuben C. A., \& Mendola P. (2010). Participation in activities outside of school hours in relation to problem behavior and social skills in middle childhood. Journal of School Health, 80(3), 119-125.

Kumpulainen, K., Rãsãnen, E., Henttonem, I., Moilanem, I., Piha, J., Puura, K., \& Tamminen, T. (1999). Children's behavioural / emotional problems: A comparison of parents' and teachers' reports for elementary school-aged children. European Child \& Adolescent Psychiatry, 8, 41-47.

Longarezi, A. M., \& Alves, T. C. (2009). A psicologia como abordagem formativa: um estudo sobre formação de professores. Revista Semestral da Associação Brasileira de Psicologia Escolar e Educacional (ABRAPEE), 13(1), 125-132.

Mariano, M. L. (2011). Análise de práticas educativas de professores na interação com alunos com e sem problemas de comportamento. Dissertação de Mestrado, Curso de Pós-Graduação em Psicologia do Desenvolvimento e Aprendizagem, Universidade Estadual Paulista, São Paulo.

Marturano, E. M, Parreira, V. L. C., \& Benzoni, S. A. G. (1997) Crianças com queixa de dificuldade escolar: avaliação das mães através da Escala Comportamental Infantil A2 de Rutter. Estudos de Psicologia, 14(3), 3-15.

Massola, G., \& Silvares, E. F. M. (1997). Percepcão do Comportamento Infantil Por Professoras Versus Sexo e Encaminhamento para Atendimento Psicológico. Psicologia. Teoria e Pesquisa, 13(3), 303-309.

Oliveira, E. T., \& Wechsler, S. M. (2002). Variáveis que afetam a aprendizagem: percepção de alunos de licenciatura e professores. Psicologia Escolar e Educacional, 6(2), 133-139.

Paiva, M. L. M. F., \& Del Prette, Z. A. P. (2009). Crenças docentes 
e implicações para o processo de ensino-aprendizagem. Revista Semestral da Associação Brasileira de Psicologia Escolar e Educacional (ABRAPEE), 13(1), 75-85.

Patterson, G. R., DeBaryshe, B. D., \& Ramsey, E. (1989). A developmental perspective on antisocial behavior. American Psychologis, 44(2), 329-335.

Patterson, G., Reid, J., \& Dishion, T. (2002). Antisocial boys. Comportamento anti-social. Santo André, SP: ESETec Editores Associados.

Pierson, M. R., Carter, E. W., Lane, K. L., \& Glaeser, B. C. (2004). Factors Influencing the Self-Determination of Transition-Age Youth With High-Incidence Disabilities. Journal of Early Childhood Research, 2(3), 273-299.

Reis, M. G. F., \& Camargo, D. M. P. (2008). Práticas escolares e desempenho acadêmico de alunos com TDAH. Revista Semestral da Associação Brasileira de Psicologia Escolar e Educacional (ABRAPEE), 12(1), 89-100.

Rosa, L. T. B, Garcia, R. M, Domingos, N. A. M., \& Silvares, E. F. M. (2001). Caracterização do Atendimento Psicológico Prestado Por um Serviço de Psicologia a Crianças com Dificuldades Escolares. Estudos de Psicologia, 17(13), 5-15.

Satake, H., Yoshida, K., Yamashita, H., Kinukawa, N., \& Takagishi, T. (2003). Agreement between parents and teachers on behavioral/ emotional problems in japanese school children using the Child Behavior Checklist. Child Psychatry and Human Development, 34, 111-126.

Uysal, A., \& Ergenekon, Y. (2010). Social skills instruction carried out by teachers working at private special education institutions in Turkey. Education and training in autism and developmental disabilities, 45(3), 459-466.

Vila, E. M. (2005). Treinamento de Habilidades Sociais em grupo com professores de crianças com dificuldade de aprendizagem: uma análise sobre procedimentos e efeito da intervenção. Dissertação de Mestrado, Universidade Federal de São Carlos, São Paulo.

Weber, L. N. D., Prado, P. M., Viezzer, A. P., \& Brandenburg, O. J. (2004). Identificação de estilos parentais: o ponto de vista dos pais e dos filhos. Psicologia: Reflexão e Crítica, 17(3), 323-331.

Webster-Stratton, C. M. Reid, J., \& Stoolmiller, M (2008). Preventing conduct problems and improving school readiness: evaluation of the Incredible Years Teacher and Child Training Programs in highrisk schools. Journal of Child Psychology and Psychiatry, 49(5), 471-488.

Zanotto, M. L. B. (1997). Formação de Professores: a contribuição da Análise Comportamental a partir da visão skinneriana de ensino. Tese de Doutorado, Pontifícia Universidade Católica de São Paulo, São Paulo.

\section{Sobre as autoras}

Alessandra Turini Bolsoni-Silva (bolsoni@fc.unesp.br) Universidade Estadual Paulista. Livre Docente

Maria Luiza Mariano (maluizamariano@yahoo.com.br) Mestre em Psicologia do Desenvolvimento e Aprendizagem Endereço: Avenida Pinheiro Machado, 2-45, Bauru-SP- 17060810

Sonia Regina Loureiro (srlourei@fmrp.usp.br)

Universidade de São Paulo. Doutora.

Endereço: R.Anita Garibaldi, 1419-Ribeirão Preto-SP-14085-480

Caroline Bonaccorsi (carol.bonaccorsi@yahoo.com.br)

Universidade Estadual Paulista - SP. Psicóloga

Endereço: Rua Sérvio Túlio Carrijo Coube, 3-33, Bauru- SP- 17012-632 